\title{
Non-alcoholic fatty liver disease and impaired proinsulin conversion as newly identified predictors of the long-term non-response to a lifestyle intervention for diabetes prevention: results from the TULIP study
}

\author{
Vera Schmid $^{1,2}$ - Robert Wagner ${ }^{1,3,4}$ - Corinna Sailer ${ }^{1,3,4}$ - Louise Fritsche ${ }^{1,3,4}$. \\ Konstantinos Kantartzis ${ }^{1,3,4}$. Andreas Peter ${ }^{1,3,4}$ - Martin Heni ${ }^{1,3,4}$. \\ Hans-Ulrich Häring ${ }^{1,3,4}$ • Norbert Stefan ${ }^{1,3,4}$. Andreas Fritsche ${ }^{1,3,4}$
}

Received: 11 April 2017 / Accepted: 11 July 2017 / Published online: 24 August 2017

(C) Springer-Verlag GmbH Germany 2017

\begin{abstract}
Aims/hypothesis Lifestyle intervention is effective to prevent type 2 diabetes. However, a considerable long-term nonresponse occurs to a standard lifestyle intervention. We investigated which risk phenotypes at baseline and their changes during the lifestyle intervention predict long-term glycaemic non-response to the intervention.

Methods Of 300 participants at high risk for type 2 diabetes who participated in a 24 month lifestyle intervention with diet modification and increased physical activity, 190 participants could be re-examined after $8.7 \pm 1.6$ years. All individuals underwent a five-point $75 \mathrm{~g}$ OGTT and measurements of body fat compartments and liver fat content with MRI and spectroscopy at baseline, 9 and 24 months during the lifestyle intervention, and at long-term follow-up. Fasting proinsulin to insulin conversion (PI/I ratio) and insulin sensitivity and secretion were calculated from the OGTT. Non-response to lifestyle
\end{abstract}

Electronic supplementary material The online version of this article (doi:10.1007/s00125-017-4407-z) contains peer-reviewed but unedited supplementary material, which is available to authorised users.

Andreas Fritsche

andreas.fritsche@med.uni-tuebingen.de

1 Department of Internal Medicine IV, University Hospital of Tübingen, Otfried-Müller-Str. 10, 72076 Tübingen, Germany

2 International Research Training Group 1302, University of Tübingen, Otfried-Müller-Str. 10, 72076 Tübingen, Germany

3 Institute for Diabetes Research and Metabolic Diseases (IDM) of the Helmholtz Centre Munich at the University of Tübingen, Tübingen, Germany

4 German Centre for Diabetes Research (DZD), Tübingen, Germany intervention was defined as no decrease in glycaemia, i.e. no decrease in AUC for glucose at 0-120 min during OGTT (AUCglucose ${ }_{0-120 \mathrm{~min}}$ ).

Results Before the lifestyle intervention, $56 \%$ of participants had normal glucose regulation and $44 \%$ individuals had impaired fasting glucose and/or impaired glucose tolerance. At long-term follow-up, $11 \%$ had developed diabetes. Multivariable regression analysis with adjustment for age, sex, BMI and change in BMI during the lifestyle intervention revealed that baseline insulin secretion and insulin sensitivity, as well as change in insulin sensitivity during the lifestyle intervention, predicted long-term glycaemic control after 9 years. In addition, increased hepatic lipid content as well as impaired fasting proinsulin conversion at baseline were newly detected phenotypes that independently predicted long-term glycaemic control.

Conclusions/interpretation Increased hepatic lipid content and impaired proinsulin conversion are new predictors, independent of change in body weight, for non-response to lifestyle intervention in addition to the confirmed factors, impaired insulin secretion and insulin sensitivity.

Keywords Fatty liver · Insulin secretion · Insulin sensitivity * Lifestyle intervention · Prediabetes · Predictors · Proinsulin

$\begin{array}{ll}\begin{array}{l}\text { Abbreviations } \\ \text { AUCglucose }_{0-120 ~ m i n}\end{array} & \begin{array}{l}\text { AUC for glucose at 0-120 min during } \\ \text { OGTT }\end{array} \\ \text { DPP } & \begin{array}{l}\text { Diabetes Prevention Program } \\ \text { Diabetes Prevention Program } \\ \text { Outcome Study }\end{array} \\ \text { DPS } & \begin{array}{l}\text { Diabetes Prevention Study } \\ \end{array}\end{array}$




$\begin{array}{ll}{ }^{1} \text { H-MRS } & \begin{array}{l}\text { Proton magnetic resonance } \\ \text { spectroscopy }\end{array} \\ \text { IFG } & \text { Impaired fasting glucose } \\ \text { IGT } & \text { Impaired glucose tolerance } \\ \text { IGI } & \text { Insulinogenic index } \\ \text { ISI } & \text { Insulin sensitivity index } \\ \text { NAFLD } & \text { Non-alcoholic fatty liver disease } \\ \text { PI/I ratio } & \text { Proinsulin/insulin ratio } \\ \text { SCAT } & \text { Subcutaneous adipose tissue } \\ \text { TAT } & \text { total adipose tissue } \\ \text { TULIP } & \text { Tübingen Lifestyle Intervention } \\ & \text { Program } \\ \text { VAT } & \text { Visceral adipose tissue }\end{array}$

\section{Introduction}

Lifestyle intervention has repeatedly and conclusively been shown to prevent or postpone the development of type 2 diabetes in high-risk individuals [1-9]. Long-term follow-up of several studies has also been reported [4, 6, 7]. The Finnish Diabetes Prevention Study (DPS) showed a relative diabetes risk reduction of up to $38 \%$ after 13 years of intervention, compared with a control group [7]. The Diabetes Prevention Program (DPP) study and the Da Qing Diabetes Prevention study also showed a significant reduction in diabetes risk (34$43 \%$ ) compared with the control group, lasting for 10 to 20 years after the lifestyle intervention $[4,6]$.

However, the effectiveness of a standard lifestyle intervention on glucose metabolism is highly variable, and a substantial number of participants develop diabetes [1, 3, 10]. Impaired insulin secretion as well as insulin resistance are wellestablished pathophysiological mechanisms in the development of type 2 diabetes [11-14]. Recently, we found that, besides baseline impaired insulin secretion, the presence of an insulinresistant fatty liver is an important predictor of the short-term response to a standard lifestyle intervention [15]. Sufficient data are still lacking to elucidate the contribution of fatty liver to the long-term effects of a lifestyle intervention [11].

It is well accepted that, besides baseline insulin sensitivity and insulin secretion, the changes in these variables during a lifestyle intervention may play a role in its success [16-19].

Perreault et al showed in the US Diabetes Prevention Program Outcome Study (DPPOS) that the regression from impaired fasting glucose (IFG) and/or impaired glucose tolerance (IGT) to a normal glucose regulation, which was strongly associated with improvement of BMI and insulin sensitivity during the lifestyle intervention, was strongly associated with a reduced incidence of diabetes [20,21].

It is well accepted that fasting proinsulin, proinsulin to insulin conversion (PI/I ratio) and proinsulin to C-peptide ratio are predictors of the incidence of type 2 diabetes [22-30]. It is of great interest that lifestyle intervention in individuals with
IFG and/or IGT seems to have only a slight influence on insulin secretion $[31,32]$. One aspect of beta cell dysfunction in addition to insulin secretion is the conversion of proinsulin to insulin (PI/I ratio); where the conversion is adversely affected, the ratio increases and the individual develops hyperproinsulinaemia [33]. An increased fasting PI/I ratio, indicating impaired proinsulin conversion, has been observed in individuals with IGT and type 2 diabetes [34,35]. Data on the impact of lifestyle intervention on fasting PI/I ratio are rare [36-38], but those available indicate that proinsulin conversion is improved after lifestyle intervention.

The Tübingen Lifestyle Intervention Program (TULIP; trial registration number KFO 114 [http://gepris.dfg.de/gepris/ projekt $/ 5396893$ ? language $=e n]$ ) was initiated in 2003 in a population at high risk for type 2 diabetes in Germany. All participants underwent a 24 month lifestyle intervention programme. One unique feature of the TULIP study is that participants repeatedly underwent whole-body MRI and ${ }^{1} \mathrm{H}$ magnetic resonance spectroscopy $\left({ }^{1} \mathrm{H}-\mathrm{MRS}\right)$ before the lifestyle intervention and at the follow-up visits. This allows for the first time the precise assessment of liver fat content and body fat composition (total adipose tissue [TAT], visceral adipose tissue [VAT], subcutaneous adipose tissue [SCAT]) before, during and after the lifestyle intervention [10, 39-41].

We have performed a long-term follow-up of participants from the TULIP study, with the aim of identifying the risk phenotypes at baseline and changes during the lifestyle intervention that predict the long-term glycaemic non-response to the intervention. This information is important to identify early those individuals who will probably not benefit from lifestyle intervention and offer them alternative treatment.

\section{Methods}

Participants and study design Individuals at high risk for type 2 diabetes from the southern part of Germany participated in TULIP, a prospective uncontrolled intervention study. They were included in the study when they fulfilled at least one of the following criteria: a family history of type 2 diabetes; $\mathrm{BMI}>27 \mathrm{~kg} / \mathrm{m}^{2}$; IGT (blood glucose $\geq 7.78 \mathrm{mmol} / \mathrm{l}$ ); or previous diagnosis of gestational diabetes. Exclusion criteria were the diagnosis of diabetes mellitus or the presence of a severe critical mental or physical illness. Informed written consent was obtained from all participants and the local medical ethics committee approved the protocol.

A total of 413 individuals were included. At baseline, medical history was obtained and a physical examination performed. Phenotypic characterisation was performed using a $75 \mathrm{~g}$ OGTT, whole-body MRI and ${ }^{1} \mathrm{H}-\mathrm{MRS}$, as well as a cycle exercise test (see below). These examinations were repeated at 9 and 24 months, and after the long-term follow-up period of about 9 years. 
After the baseline examination, all participants underwent 15 sessions of standardised individual face-to-face dietary counselling by trained diabetes educators. In the first 9 months of intervention, i.e. the intensive lifestyle intervention phase of the study, monthly visits were scheduled. The moderate maintenance phase of the lifestyle intervention, comprising 3monthly visits, lasted from the ninth month until the end of the second year.

Counselling aimed to reduce body weight by $\geq 5 \%$. Furthermore, participants were recommended to reduce the energy intake from fat to $<30 \%$ of total energy consumed, and to increase dietary fibre intake to at least $15 \mathrm{~g} / 4185 \mathrm{~kJ}$ $(1000 \mathrm{kcal})$. They were also encouraged to reduce the intake of saturated fatty acids to $<10 \%$ of total fat. During each visit, participants presented a 3 day food diary and discussed it with the dietitians. Diet composition was estimated with a validated software, using two representative days of the 3 day diary (DGE-PC 3.0; Deutsche Gesellschaft für Ernährung, Bonn, Germany). Individuals were motivated to perform at least $3 \mathrm{~h}$ moderate exercise per week. A heart rate monitor (Polar, Büttelbronn, Germany) was given to the participants and they were instructed to exercise so that their heart rate was just below their individual anaerobic threshold. Individual anaerobic threshold was measured by a motorised treadmill (Saturn, HP-Cosmos, Traunstein, Germany).

OGTT All individuals underwent a frequently sampled $75 \mathrm{~g}$ OGTT at baseline, after 9 months, after 24 months and at the long-term follow-up appointment. For the determination of plasma glucose and insulin levels, venous blood samples were obtained at $0,30,60,90$ and $120 \mathrm{~min}$. Plasma glucose was measured using a bedside glucose analyser (YSI, Yellow Springs, CO, USA). AUC for glucose at 0-120 min during OGTT (AUCglucose ${ }_{0-120 ~ m i n}$ ) was calculated with the trapezoid method. Plasma insulin and C-peptide were determined by an immunoassay with the ADVIA Centaur XP Immunoassay System (Siemens Healthcare Diagnostics, Erlangen, Germany).

Serum proinsulin concentrations were measured using a microparticle enzyme immunoassay (IBL, Hamburg, Germany) on a BEP III System (Siemens Healthcare, Erlangen, Germany). The proinsulin assay measures intact proinsulin and has $0 \%$ cross-reactivity with human insulin and C-peptide. There is cross-reactivity with the relevant proinsulin split products: des-31,32-proinsulin, 0.9\%; des-32,33proinsulin, $1.5 \%$; des-64,65-proinsulin, 53\%; and des-65,66proinsulin, 55\% [42]. Insulin sensitivity was calculated from glucose and insulin values during the OGTT as proposed by Matsuda and DeFronzo (i.e. to calculate the insulin sensitivity index [ISI]) [43]. Insulin secretion was estimated using the insulinogenic index (IGI) across $30 \mathrm{~min}\left(\mathrm{IGI}_{0-30 \mathrm{~min}}\right)$, calculated as $\mathrm{Ins}_{30}-\mathrm{Ins}_{0} / \mathrm{Glc}_{30}-\mathrm{Glc}_{0}$, where $\mathrm{Ins}_{0}$ and $\mathrm{Glc}_{0}$ is insulin and glucose at $0 \mathrm{~min}$, and $\mathrm{Ins}_{30}$ and $\mathrm{Glc}_{30}$ is insulin and glucose at $30 \mathrm{~min}$ [17]. The relative insulin secretion (disposition index), which describes the insulin secretion relative to insulin sensitivity, was calculated as ISI $\times \operatorname{IGI}_{0-30} \min [17]$. Fasting proinsulin to insulin conversion was determined by dividing proinsulin $n_{0 \text { min }}$ by insulin $_{0}$ min $(\mathrm{PI} / \mathrm{I}$ ratio), where proinsulin $_{0 \text { min }}$ is proinsulin at $0 \mathrm{~min}$ and insulin ${ }_{0 \text { min }}$ is insulin at $0 \mathrm{~min}$. Fasting proinsulin to $\mathrm{C}$-peptide ratio was determined by dividing proinsulin 0 min by $\mathrm{C}$-peptide at $0 \mathrm{~min}(\mathrm{C}$ peptide $_{0 \mathrm{~min}}$ ) [28].

T1-weighted MRI to measure body fat Determination of whole-body fat distribution was performed using an axial T1-weighted fast spin echo technique as previously described using a 1.5 T whole-body imager (Magnetom Sonata, Siemens Medical Solutions, Erlangen, Germany) [14, 40].

${ }^{1} \mathrm{H}-\mathrm{MRS}$ to measure intrahepatic fat content Liver fat content was quantified by localised ${ }^{1} \mathrm{H}-\mathrm{MRS}$ as previously described [14, 40]. Non-alcoholic fatty liver disease (NAFLD) was defined as liver fat content $\geq 5.56 \%$ [44].

Maximal oxygen consumption The individuals underwent a continuous, incremental exercise test on a cycle ergometer to volitional exhaustion. The cycle ergometer test was performed on an electromagnetically braked cycle ergometer (Ergometrics 800 S; Ergoline, Bitz, Germany). Oxygen consumption was measured using spiroergometry (Cortex MetaLyzer, Metasoft 3.9, Leipzig, Germany). Maximal oxygen consumption $\left(\dot{V} \mathrm{O}_{2 \max }\right)$ is expressed as $\dot{V} \mathrm{O}_{2 \max }$ per kg lean body mass $\left(\mathrm{ml} \mathrm{min}^{-1} \mathrm{~kg}^{-1}\right)$.

Statistical analyses The primary endpoint of the study was change in $\mathrm{AUC}_{\text {glucose0-120 min }}$ (response/non-response to lifestyle intervention). Data are given as mean $\pm \mathrm{SE}$. A Wilcoxonsigned-rank test was used to compare variables before and after lifestyle intervention and during long-term follow-up. Prior to multivariable regression analyses, variable distributions were tested for normality using the Shapiro-Wilk test. Non-normally distributed variables were transformed to their natural logarithm. To identify key model determinants, forward stepwise multivariable linear regression analyses were performed. A $p$ value $\leq 0.05$ was considered statistically significant. The software package JMP 11 (SAS Institute, Cary, $\mathrm{NC}$, USA) was used for all other analyses.

\section{Results}

Longitudinal results A total of 413 participants were enrolled in the study, with 357 undertaking 9 months of intensive lifestyle intervention, and 300 completing the full intervention in the 24 month study after (Fig. 1). We invited these 300 participants, via written invitation or telephone call, to a 


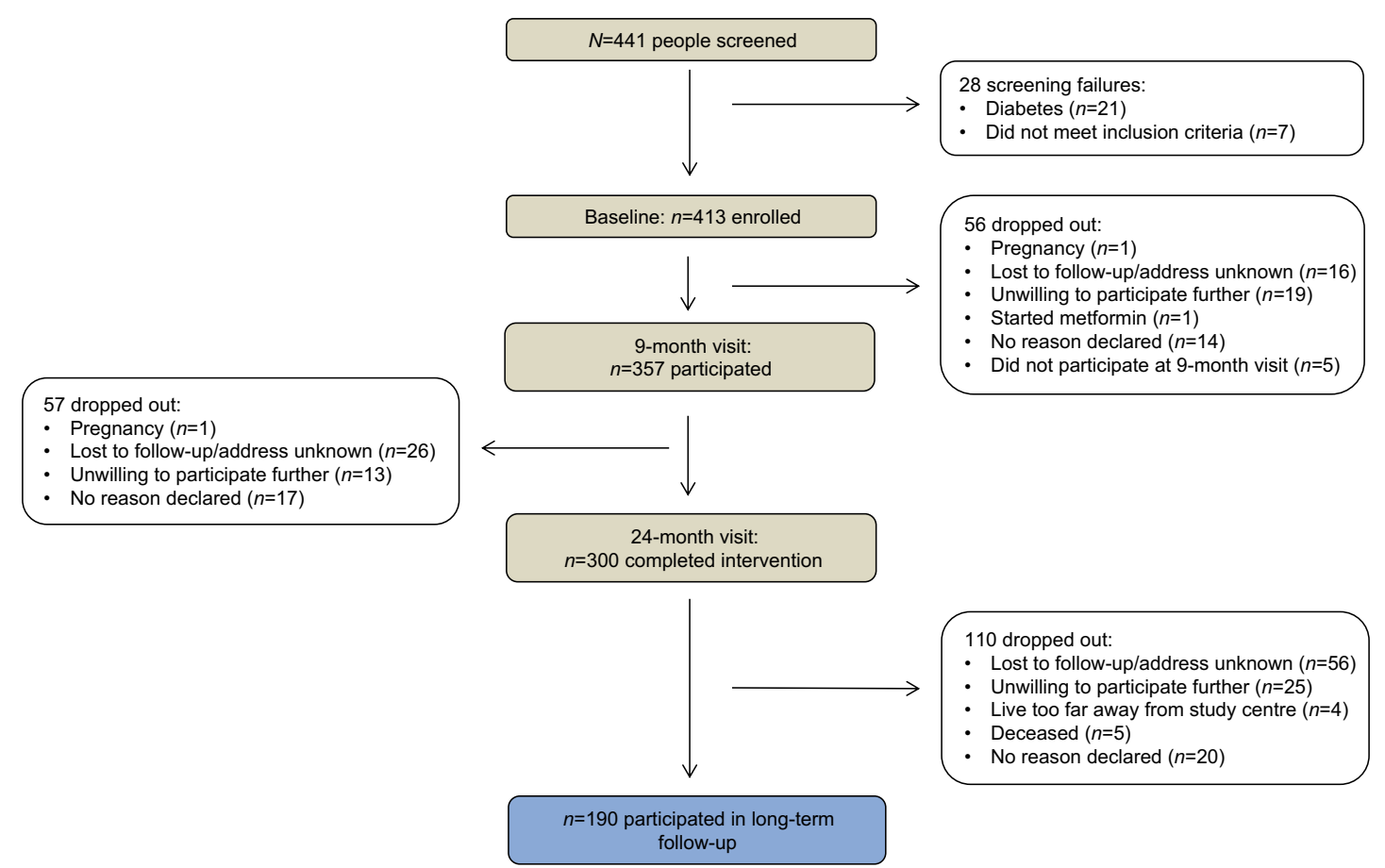

Fig. 1 Trial profile of the TULIP study: screening, enrolment and post-intervention follow-up period ( $8.7 \pm 1.6$ years)

long-term follow-up visit; 190 individuals $(63.3 \%)$ attended after a mean total follow-up time (time between baseline and long-term follow-up) of $8.7 \pm 1.6$ years (Fig. 1). The main reason for not participating in the long-term follow-up was unknown address or loss to follow-up (Fig. 1). Table 1 shows the participant characteristics during the four visits. After 9 months of intervention, AUCglucose ${ }_{0-120}$ min values improved in $63 \%$ of the participants (responders). This ratio of responders was decreased at the long-term follow-up to $43 \%$. At the long-term follow-up, $46 \%$ of the 190 participants had a normal glucose regulation (normal glucose tolerance and normal fasting glucose), $43 \%$ participants had IFG and/or IGT and 21 participants (11\%) developed diabetes (Fig. 2).

Among the 190 participants who attended all visits, fasting and postprandial plasma glucose levels, insulin sensitivity, the disposition index, fasting proinsulin, fasting insulin and fasting PI/I ratio improved during the 9 month intensive period of the lifestyle intervention. Body weight, liver fat content, TAT, SCAT and VAT decreased (Table 2, Fig. 3).

Comparing the baseline with the long-term follow-up variables after 9 years, fasting PI/I ratio and fasting proinsulin improved from baseline to long-term follow-up (both
Table 1 Characteristics of participants at different time points

\begin{tabular}{|c|c|c|c|c|}
\hline \multirow[t]{2}{*}{ Characteristic } & \multirow[t]{2}{*}{ Baseline } & \multicolumn{2}{|c|}{ Lifestyle intervention } & \multirow{2}{*}{$\begin{array}{l}\text { Long-term } \\
\text { follow-up }\end{array}$} \\
\hline & & 9 months & 24 months & \\
\hline$n$ & 413 & 357 & 300 & 190 \\
\hline $\operatorname{Sex}(\mathrm{F} / \mathrm{M})$ & $258 / 155$ & $222 / 135$ & $182 / 118$ & $112 / 78$ \\
\hline Age (years) & $45 \pm 11$ & $47 \pm 11$ & $49 \pm 11$ & $58 \pm 10$ \\
\hline Body weight (kg) & $88.9 \pm 1.0$ & $85.5 \pm 1.0$ & $86.0 \pm 1.1$ & $86.9 \pm 1.4$ \\
\hline BMI $\left(\mathrm{kg} / \mathrm{m}^{2}\right)$ & $30.3 \pm 0.3$ & $29.2 \pm 0.3$ & $29.3 \pm 0.3$ & $30.1 \pm 0.4$ \\
\hline WHR & $0.89 \pm 0.0$ & $0.88 \pm 0.0$ & $0.89 \pm 0.0$ & $0.91 \pm 0.01$ \\
\hline Fasting blood glucose (mmol/l) & $5.2 \pm 0.0$ & $5.2 \pm 0.0$ & $5.3 \pm 0.0$ & $5.7 \pm 0.1$ \\
\hline $2 \mathrm{~h}$ blood glucose $(\mathrm{mmol} / \mathrm{l})$ & $6.9 \pm 0.1$ & $6.7 \pm 0.1$ & $7.2 \pm 0.1$ & $7.2 \pm 0.2$ \\
\hline AUCglucose $_{0-120 \text { min }}(\mathrm{mmol} \times \mathrm{h} / \mathrm{l})$ & $16.1 \pm 0.1$ & $15.6 \pm 0.2$ & $16.4 \pm 0.2$ & $16.9 \pm 0.3$ \\
\hline
\end{tabular}

Data are means $\pm \mathrm{SE}$

F, female; M, male 


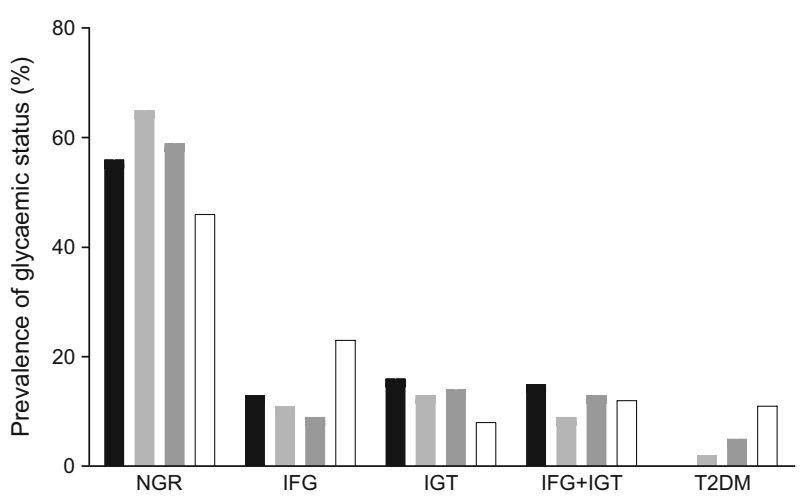

Fig. 2 Progression of glycaemic status from baseline to long-term follow-up. Data are given as percentages. $n=190$ participants. Black, baseline; light grey, 9 months of lifestyle intervention; dark grey, 24 months of lifestyle intervention; white, long-term follow-up. NGR, normal glucose regulation; T2DM, type 2 diabetes mellitus

$p \leq 0.0001)$, while post-challenge $(2 \mathrm{~h})$ plasma glucose, AUCglucose $_{0-120}$ min during the OGTT and liver fat content did not change significantly. In contrast, fasting glucose levels increased and insulin sensitivity, as well as the disposition index, decreased significantly. Furthermore, body weight, TAT, SCAT, VAT and fasting insulin increased with the concomitant deterioration of cardiorespiratory fitness during this period (all $p \leq 0.05$, Table 2, Fig. 3).

\section{Impact of body weight change during the first 9 months of} lifestyle intervention on long-term success Decrease in body weight during the lifestyle intervention was found to associate with the success of the lifestyle intervention to improve glycaemia $[3,4,16]$. We therefore performed multivariable linear regression analysis with change in BMI during the intensive period of the lifestyle intervention as effect variable and AUCglucose ${ }_{0-120 \text { min }}$ levels at long-term follow-up as the dependent variable. The model was additionally adjusted for sex, age, follow-up time, AUCglucose ${ }_{0-120}$ min and BMI at baseline. The change in BMI during the intensive period of the lifestyle intervention was associated with the AUCglucose ${ }_{0-120}$ min level in long-term follow-up ( $\beta=0.206, p=0.0006$; Table 3, model 1).

\section{Impact of insulin sensitivity and insulin secretion on long-} term success of the lifestyle intervention Insulin secretion and insulin sensitivity are important pathophysiological factors in the pathogenesis of type 2 diabetes [11]. Therefore, these variables and the change of both during the intensive phase of lifestyle intervention were added to model 1. As shown in Table 3 (model 2), the baseline insulin secretion $(\beta=-0.436, p \leq 0.0001)$ and insulin sensitivity $(\beta=-0.405, p=0.0009)$ and the change in insulin sensitivity ( $\beta=-0.230, p=0.0015)$, were associated with the AUCglucose $_{0-120}$ min level at the long-term follow-up.

To visualise the effect of baseline insulin secretion on the cumulative probability of not developing diabetes, Kaplan-
Meier survival curves were generated. Not a single diabetes case was diagnosed in individuals with a low disposition index compared with a high disposition index at baseline (Fig. 4a).

Impact of hepatic lipid content on the long-term success of the lifestyle intervention In a next step, hepatic lipid content at baseline and change in content during the intensive phase of the lifestyle intervention were added to the model (Table 3, model 3). Hepatic lipid content at baseline ( $\beta=0.231$, $p=0.0207)$ predicted AUCglucose $\mathrm{e}_{0-120 \mathrm{~min}}$ at long-term follow-up, in addition to baseline insulin secretion, change in insulin sensitivity and age.

To visualise the effect of baseline hepatic lipid content on the cumulative probability of not developing diabetes, Kaplan-Meier survival curves were generated. In participants with or without NAFLD, individuals without NAFLD at baseline had a significantly lower risk for developing diabetes compared with individuals with NAFLD (logrank test, $p=0.0094)$ (Fig. 4b).

Impact of fasting proinsulin to insulin conversion on the long-term success of the lifestyle intervention Proinsulin conversion has been shown to have an impact on diabetes risk $[34,35]$. Therefore, the fasting PI/I ratio at baseline and the change in the fasting PI/I ratio during the intensive phase of the lifestyle intervention were added to model 3 . The baseline fasting PI/I ratio predicted the long-term AUCglucose ${ }_{0-120 ~ m i n}$ ( $\beta=0.204, p=0.0444)$, in addition to the baseline insulin secretion and the change in insulin sensitivity during the intensive phase of the lifestyle intervention. There is an inverse correlation of fasting PI/I ratio and hepatic lipid content ( $\beta=-0.208, p=0.0213$ ), thus, the impact of liver fat was no longer significant in this model. Of note, model 4 gave the highest $r^{2}$ value for glucose levels at long-term follow-up, explaining $59 \%$ of variability in AUCglucose $\mathrm{A}_{0-120} \mathrm{~min}$. Furthermore, the ratio of proinsulin to C-peptide impacts similarly on long-term glycaemia as the $\mathrm{PI} / \mathrm{I}$ ratio (see electronic supplementary material [ESM] Table 1).

\section{Discussion}

This long-term follow-up analysis of a standard lifestyle intervention programme aiming at preventing type 2 diabetes provides three major results: first, we show, for the first time, data on the long-term development of body fat composition and ectopic fat deposition in the liver, together with changes in insulin sensitivity and insulin secretion following a lifestyle intervention. Second, we show that the long-term success in maintaining glycaemia can be inferred from baseline variables and short-term response to the lifestyle intervention. Third, we identified a baseline high-risk phenotype for the long-term glycaemic non-response that includes, besides impaired 
Table 2 Anthropometric and metabolic profile of 190 participants from baseline to the long-term follow-up

\begin{tabular}{|c|c|c|c|c|c|c|c|}
\hline \multirow[t]{2}{*}{ Characteristic } & \multirow[t]{2}{*}{ Baseline } & \multicolumn{2}{|c|}{ Lifestyle intervention } & \multirow{2}{*}{$\begin{array}{l}\text { Long-term } \\
\text { follow-up }\end{array}$} & \multicolumn{3}{|l|}{$p$ value } \\
\hline & & 9 months & 24 months & & $\begin{array}{l}\text { Baseline vs } \\
9 \text { months' } \\
\text { intervention }\end{array}$ & $\begin{array}{l}\text { Baseline vs } \\
24 \text { months' } \\
\text { intervention }\end{array}$ & $\begin{array}{l}\text { Baseline vs } \\
\text { long-term } \\
\text { follow-up }\end{array}$ \\
\hline $\operatorname{Sex}(f / m)$ & $112 / 78$ & & & & & & \\
\hline Age (years) & $49 \pm 10$ & $49 \pm 10$ & $51 \pm 10$ & $58 \pm 10$ & & & \\
\hline Body weight $(\mathrm{kg})$ & $86.3 \pm 1.3$ & $83.3 \pm 1.2$ & $84.6 \pm 1.3$ & $86.9 \pm 1.4$ & $<0.0001$ & $<0.0001$ & 0.3917 \\
\hline Body height $(\mathrm{cm})$ & $170.4 \pm 0.7$ & $170.5 \pm 0.7$ & $170.4 \pm 0.7$ & $169.7 \pm 0.7$ & 0.2500 & 0.5093 & $<0.0001$ \\
\hline BMI $\left(\mathrm{kg} / \mathrm{m}^{2}\right)$ & $29.7 \pm 0.4$ & $28.6 \pm 0.4$ & $29.1 \pm 0.4$ & $30.1 \pm 0.4$ & $<0.0001$ & 0.0001 & 0.0211 \\
\hline WHR & $0.9 \pm 0.01$ & $0.89 \pm 0.01$ & $0.89 \pm 0.01$ & $0.91 \pm 0.01$ & $<0.0001$ & 0.0115 & 0.0043 \\
\hline Fasting blood glucose $(\mathrm{mmol} / \mathrm{l})$ & $5.3 \pm 0.0$ & $5.2 \pm 0.0$ & $5.3 \pm 0.0$ & $5.7 \pm 0.1$ & 0.0171 & 0.5365 & $<0.0001$ \\
\hline $2 \mathrm{~h}$ blood glucose $(\mathrm{mmol} / \mathrm{l})$ & $7.1 \pm 0.1$ & $6.8 \pm 0.1$ & $7.3 \pm 0.1$ & $7.2 \pm 0.2$ & 0.0136 & 0.5654 & 0.4441 \\
\hline $\begin{array}{l}\text { AUCglucose }_{0-120 ~ \min } \\
(\mathrm{mmol} \times \mathrm{h} / \mathrm{l})\end{array}$ & $16.6 \pm 0.2$ & $15.9 \pm 0.2$ & $16.6 \pm 0.2$ & $16.9 \pm 0.3$ & 0.0004 & 0.9425 & 0.1771 \\
\hline Fasting insulin (pmol/1) & $65.21 \pm 3.38$ & $55.30 \pm 2.72$ & $61.46 \pm 2.78$ & $101.95 \pm 4.44$ & 0.0006 & 0.1806 & $<0.0001$ \\
\hline Fasting proinsulin (pmol/l) & $5.86 \pm 0.56$ & $4.02 \pm 0.45$ & $3.42 \pm 0.26$ & $3.24 \pm 0.21$ & 0.0020 & 0.0001 & $<0.0001$ \\
\hline $\begin{array}{l}\text { Fasting proinsulin conversion } \\
(\mathrm{PI} / \mathrm{I} \text { ratio })^{\mathrm{a}}\end{array}$ & $0.113 \pm 0.01$ & $0.085 \pm 0.01$ & $0.071 \pm 0.01$ & $0.038 \pm 0.00$ & 0.0139 & 0.0208 & $<0.0001$ \\
\hline Fasting C-peptide (pmol/l) & $613 \pm 20$ & $553 \pm 16$ & $583 \pm 19$ & $616 \pm 18$ & $<0.0001$ & 0.0567 & 0.8946 \\
\hline $\begin{array}{l}\text { Fasting proinsulin to C-peptide } \\
\text { ratio }\end{array}$ & $0.011 \pm 0.001$ & $0.008 \pm 0.011$ & $0.006 \pm 0.001$ & $0.007 \pm 0.001$ & 0.0089 & 0.0123 & 0.0002 \\
\hline Insulin sensitivity & $12.1 \pm 0.5$ & $13.8 \pm 0.6$ & $12.6 \pm 0.5$ & $8.4 \pm 0.4$ & 0.0001 & 0.7301 & 0.0001 \\
\hline $\mathrm{IGI}_{0-30 \mathrm{~min}}$ & $133 \pm 8$ & $134 \pm 8$ & $136 \pm 10$ & $163 \pm 8$ & 0.3948 & 0.3741 & $<0.0001$ \\
\hline $\begin{array}{l}\text { Insulin secretion } \\
\text { (disposition index) }\end{array}$ & $1411 \pm 113$ & $1655 \pm 126$ & $1458 \pm 132$ & $1169 \pm 77$ & 0.0001 & 0.0836 & $<0.0001$ \\
\hline$\dot{V} \mathrm{O}_{2 \max }\left(\mathrm{ml} \mathrm{min} \mathrm{mg}^{-1} \mathrm{~kg}^{-1}\right)^{\mathrm{b}}$ & $26.5 \pm 0.7$ & $27.2 \pm 0.7$ & $26.4 \pm 0.8$ & $21.0 \pm 0.6$ & 0.0548 & 0.8802 & $<0.0001$ \\
\hline Hepatic lipid content $(\%)^{\mathrm{c}}$ & $6.0 \pm 0.7$ & $4.1 \pm 0.4$ & $4.8 \pm 0.5$ & $6.1 \pm 0.6$ & $<0.0001$ & 0.0031 & 0.5452 \\
\hline TAT $(1)^{\mathrm{d}}$ & $30.4 \pm 1.1$ & $27.9 \pm 1.0$ & $29.2 \pm 1.1$ & $34.9 \pm 1.1$ & $<0.0001$ & 0.0105 & $<0.0001$ \\
\hline $\operatorname{VAT}(1)^{\mathrm{e}}$ & $3.5 \pm 0.2$ & $2.9 \pm 0.2$ & $3.2 \pm 0.2$ & $4.8 \pm 0.3$ & $<0.0001$ & 0.0003 & $<0.0001$ \\
\hline $\operatorname{SCAT}(1)^{\mathrm{f}}$ & $9.0 \pm 0.5$ & $7.9 \pm 0.4$ & $6.8 \pm 0.4$ & $12.6 \pm 0.6$ & $<0.0001$ & $<0.0001$ & $<0.0001$ \\
\hline
\end{tabular}

Data are means \pm SE; data were available for the following numbers of participants: ${ }^{\mathrm{a}} 151 ;{ }^{\mathrm{b}} 100 ;{ }^{\mathrm{c}} 99 ;{ }^{\mathrm{d}} 104 ;{ }^{\mathrm{e}} 106 ;{ }^{\mathrm{f}} 93$

Liver fat content was measured by ${ }^{1} \mathrm{H}$-MRS. A Wilcoxon-signed-rank test was used to compare variables before and after lifestyle intervention and at long-term follow-up

insulin secretion and insulin sensitivity, fatty liver or impaired fasting proinsulin conversion.

Recent studies have shown that a lifestyle intervention is effective in preventing or delaying the development of type 2 diabetes during both short- and long-term follow-up [3-7, 45, 46]. The lifestyle changes comprised several beneficial changes in diet, as well as increased physical activity, with beneficial effects on blood glucose levels and a significant reduction in diabetes incidence $[3,45]$. The TULIP intervention was most intensive during the first 9 months. Consequently, the benefit was most prominent during this time. Body weight reduction during the intensive period was moderate, as observed in two other studies including individuals with IGT $[3,45]$.

Using whole-body MRI, we observed a marked reduction in major body fat compartments (TAT, VAT and SCAT) between $10 \%$ and $15 \%$ during the first 9 months of intensive intervention. Importantly, liver fat content improved by more than $30 \%$ during that period. This 'qualified' weight reduction with decreases in visceral fat mass and liver fat content improved insulin sensitivity by nearly $15 \%$. These risk factors are known to be associated with metabolically unhealthy obesity [47]. VAT and intrahepatic fat are the source of several hormones and cytokines that act against insulin and initiate a chronic systemic activation of the immune system. This chronic systemic inflammation is the key driver for further impairment of insulin resistance and also for the chronic progressive nature of the disease [48]. Lifestyle interventions can only modulate disease development if there is an impact on the hormonal activity of the body fat compartments and not just on the volume of body fat [49].

In contrast to the favourable short-term effects of a lifestyle intervention, the improvements in metabolic variables are lost over time when body weight and body fat composition, as 
a

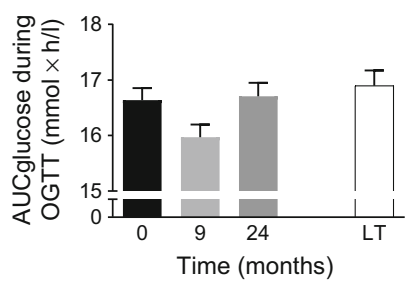

C
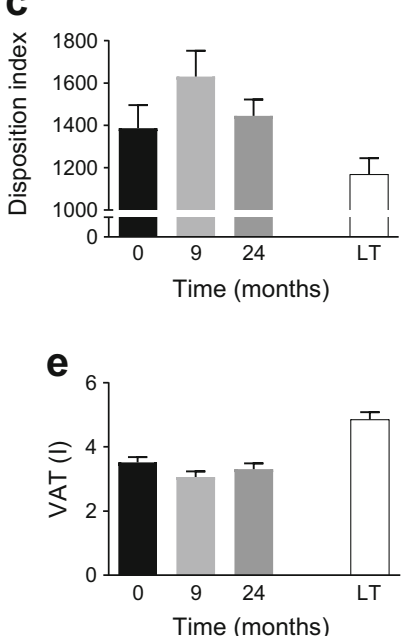

b
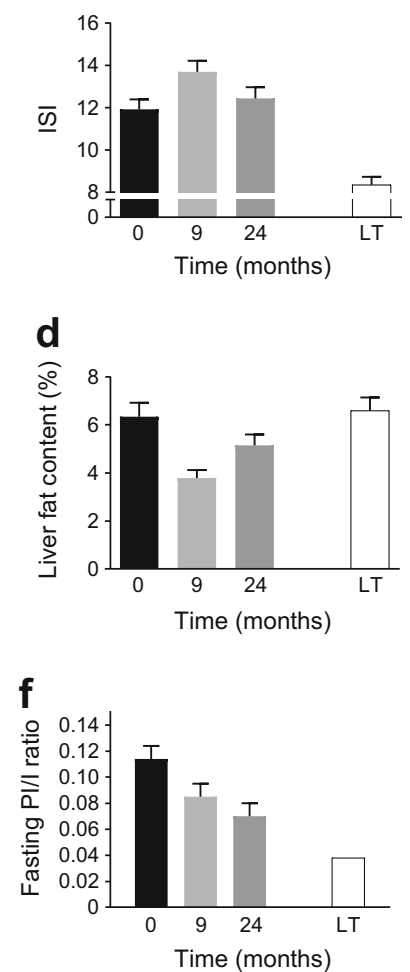

Fig. 3 Progression of metabolic variables and fat compartments from baseline to the long-term follow-up. Data are given as mean $\pm \mathrm{SE}$. AUCglucose $_{0-120 ~ m i n}$ during OGTT (a), ISI (b), insulin secretion (disposition index [ISI $\left.\left.\times \mathrm{IGI}_{0-30 \mathrm{~min}}\right]\right)(\mathbf{c})$, liver fat content $(\mathbf{d})$, VAT $(\mathbf{e})$ and fasting PI/I ratio (f) at baseline, after 9 months and 24 months of lifestyle intervention and at long-term follow-up; LT, long-term follow-up

well as insulin sensitivity and insulin secretion, deteriorate. Despite gain in body weight and body fat, postprandial blood glucose levels and liver fat content were comparable with baseline levels, even after 9 years (Table 2, Fig. 3). One important variable that improved during the lifestyle intervention and was improved at long-term follow-up was fasting proinsulin conversion. As fasting insulin was doubled at long-term follow-up compared with baseline and C-peptide was unchanged, this indicates declining hepatic insulin clearance over this time (9 years), suggesting improved proinsulin conversion. Fasting proinsulin levels also decreased by $\sim 50 \%$, also indicating that proinsulin conversion was improved.

In addition, it should be highlighted that a rate of $11 \%$ incident diabetes is rather low compared with similar lifestyle intervention programmes. In the lifestyle intervention group of the Finnish DPS and the US DPPOS, 29\% and $40 \%$ of participants, respectively, developed diabetes during the long-term follow-up $[4,7]$. This might in part be because both studies exclusively included participants with IGT at baseline, while the TULIP study included a metabolically healthier cohort. Taken together, our results are in line with earlier studies showing that healthy changes in diet and increased exercise can have beneficial long- term effects on glycaemia, and offer encouraging evidence for the effectiveness of a lifestyle intervention, even without a large reduction in body weight $[4,6,7,20]$.

Nevertheless, the problem of non-response with respect to an improvement of glycaemia during the lifestyle intervention in a substantial number of participants remains $[4,6,7,20]$. It is, therefore, clinically important to define risk factors and risk phenotypes associated with such a non-response.

One factor might be the decrease in insulin sensitivity that is correlated with the amount of weight loss [19]. However, the correlation of weight loss and glycaemia is not very strong [15]. Paradoxically, in the US DPPOS a stronger weight loss even adversely affected diabetes risk, independent of the previous intervention [20]. Thus, other metabolic determinants may additionally affect the long-term effectiveness of a lifestyle intervention. In the present study, we addressed the pathophysiological determinants that predict non-response to the lifestyle intervention. We were able to show that low insulin secretion capacity at baseline, low insulin sensitivity and impaired improvement in insulin sensitivity during the intensive first 9 months of the lifestyle intervention predicted nonresponse to the intervention. These findings accord with previous studies that have shown that both baseline insulin secretion and insulin sensitivity, as well as the change thereof during a lifestyle intervention, are predictors of diabetes development $[16,19,50]$ or, vice versa, regression to normal glucose tolerance $[20,21]$.

A new finding was that a high liver fat content at baseline or an impaired fasting relative proinsulin to insulin conversion at baseline were important determinants of impaired glycaemia at long-term follow-up, independent of insulin secretion and insulin sensitivity. Models containing these pathophysiological factors provided a better prediction $\left(r^{2}=0.55\right.$ and 0.59 , respectively) of long-term glycaemia than a simpler model containing baseline BMI as well as change in BMI $\left(r^{2}=0.45\right)$ (Table 3$)$.

The conversion of proinsulin to insulin is thought to be a biomarker for beta cell function [26, 51, 52]. It is important that in the present study proinsulin to insulin conversion predicted long-term glycaemia after the lifestyle intervention, independently of insulin secretion and insulin sensitivity (model 4). Our results are in line with earlier studies that showed that increased proinsulin levels, which are the consequence of impaired proinsulin conversion to insulin, predict worsening of glucose tolerance status [53, 54]. Furthermore, it has been shown that fasting proinsulin conversion and the acute insulin response independently impact on the development of type 2 diabetes [27, 55] highlighting that there are multiple aspects to beta cell dysfunction. In this context, the improvement of proinsulin to insulin conversion after the lifestyle intervention and at the long-term follow-up is an important finding. One may speculate that proinsulin conversion is a useful biomarker for both the prediction of diabetes risk and monitoring the success of the lifestyle intervention. 


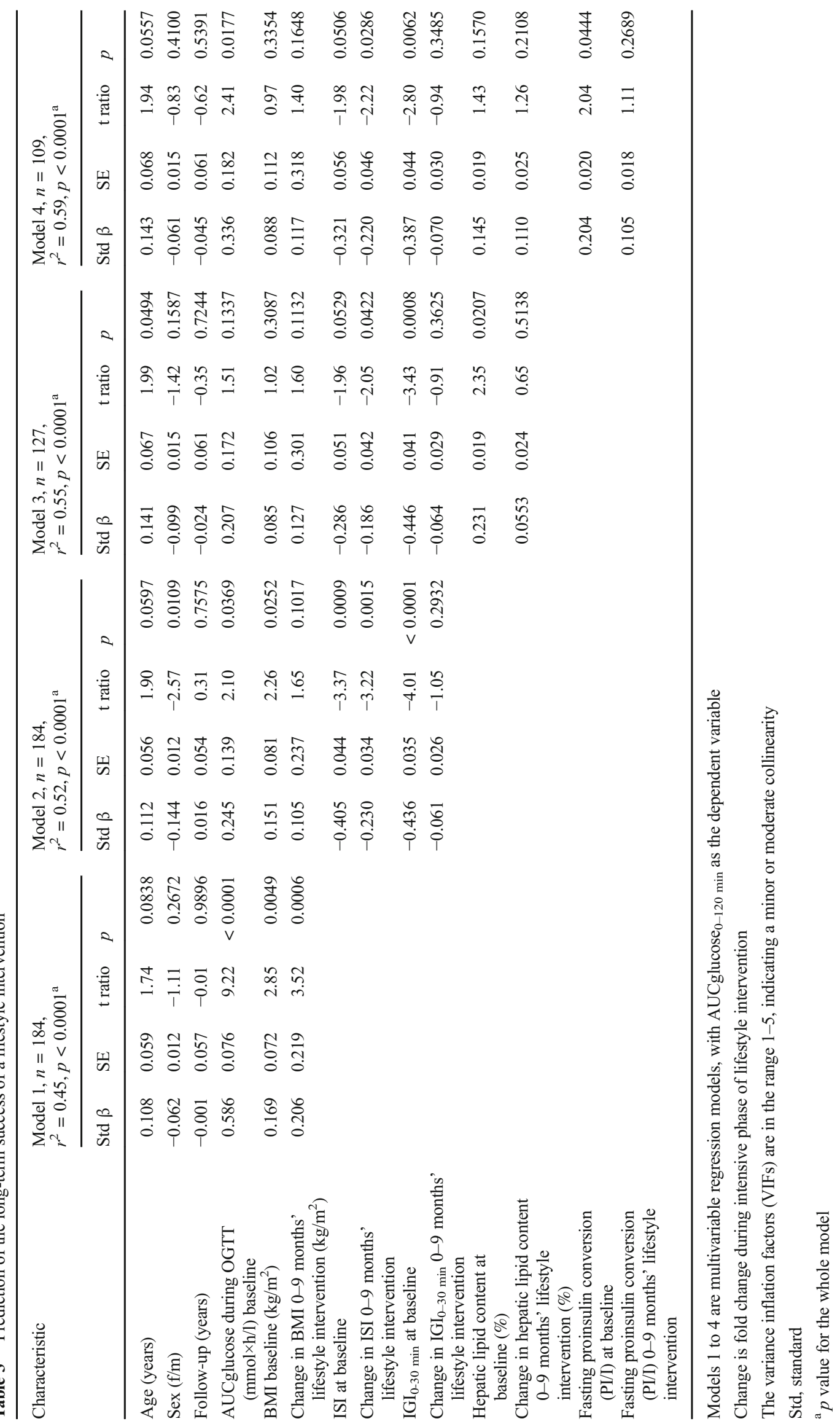




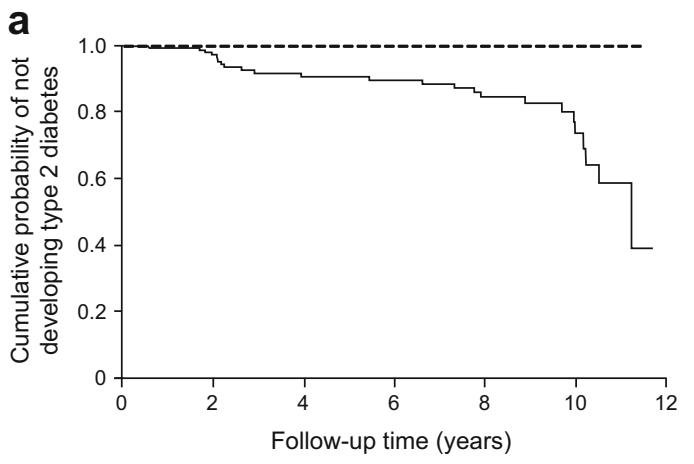

Fig. 4 Cumulative probability of not developing type 2 diabetes and prediction models. (a) Cumulative probability of not developing type 2 diabetes in the TULIP study in a group with a high (dashed line) or low (solid line) disposition index at baseline, according to whether they were

For the design, development and practice of improved and more successful lifestyle intervention programmes, it is important to reveal risk factors for short- and long-term success of a lifestyle intervention. Regarding short-term success, we previously found that the phenotype encompassing participants with a low disposition index or insulin-resistant NAFLD have a high risk for acute glycaemic non-response to lifestyle intervention [15]. The present study now demonstrates that such a phenotype is also associated with long-term non-response and extends the phenotype to impaired proinsulin conversion. Individuals with such a high-risk phenotype may be put on a more intensive lifestyle intervention at an earlier stage, or even receive pharmacological therapy.

One limitation of the present analysis is that the TULIP study was designed as an uncontrolled study. Therefore, we cannot compare the risk of non-response to the lifestyle intervention (i.e. improvement in glycaemia) with the risk of developing diabetes without intervention. However, a lifestyle intervention is standard care in individuals at high risk for type 2 diabetes [56] after the publication of the landmark studies, the DPS and DPP, and thus, a non-treated control group seems inappropriate. Furthermore, our study was conducted at a single academic research centre and the results cannot probably be generalised to other populations.

In conclusion, we found that, independent of the important change in body weight, an impaired insulin secretion, a fatty liver or an impaired fasting proinsulin conversion before lifestyle intervention predicted glycaemic non-response to the lifestyle intervention programme. Our current results may help to develop individualised prevention programmes targeting those with at-risk phenotypes. Furthermore, they may also aid monitoring during such programmes and may help to identify those for whom treatment intensification will be necessary.

Acknowledgements We thank all the study participants for their cooperation with this project. We gratefully acknowledge the excellent technical assistance of A. Bury, E. Kollmar, A. Vosseler and A. Dessecker b

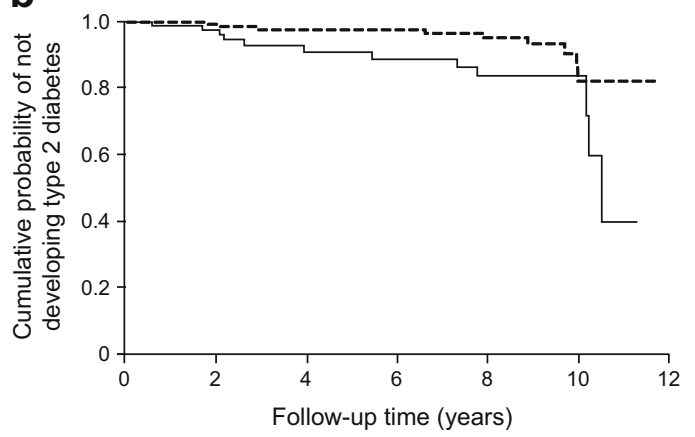

above or below the median. Logrank test, $p<0.0001$. (b) Cumulative probability of not developing type 2 diabetes in the TULIP study in a group with (solid line) or without NAFLD (dashed line) at baseline (cutoff point, $5.56 \%$ liver fat). Logrank test, $p=0.0094$

(all University of Tübingen, Germany). We further thank J. Machann and F. Schick from the Section for Experimental Radiology for the excellent body imaging and cooperation within the study.

Data availability The datasets generated during and/or analysed during the current study are not publicly available because the participants have not granted permission for this. The datasets are available from the corresponding author on reasonable request.

Funding The study was supported in part by grants from the Deutsche Forschungsgemeinschaft and the German Federal Ministry of Education and Research (BMBF) to the German Centre for Diabetes Research (DZD). The study was supported by a grant of the DZD to AF.

Duality of interest The study was initiated by the authors and there are no other sponsors other than those acknowledged. The authors declare that there is no duality of interest associated with this manuscript.

Contribution statement All authors provided substantial contributions to the conception and design, acquisition of data or analysis and interpretation of data, drafting the article or revising it critically for important intellectual content. They all gave final approval of the version to be published. The most important contributions of each author were as follows. VS and AF researched data and wrote the manuscript. RW, CS, LF, $\mathrm{KK}, \mathrm{AP}$ and $\mathrm{MH}$ researched data and edited the manuscript. AF, NS and HUH designed the study, contributed to the discussion and reviewed the manuscript. AF is the guarantor of this work and, as such, had full access to all the data in the study and takes responsibility for the integrity of the data and the accuracy of the data analysis.

\section{References}

1. Tuomilehto J, Lindström J, Eriksson JG et al (2001) Prevention of type 2 diabetes mellitus by changes in lifestyle among subjects with impaired glucose tolerance. N Engl J Med 344:1343-1350

2. Lindström J, Ilanne-Parikka P, Peltonen M et al (2006) Sustained reduction in the incidence of type 2 diabetes by lifestyle intervention: follow-up of the Finnish Diabetes Prevention Study. Lancet 368:1673-1679

3. Knowler WC, Barrett-Connor E, Fowler SE et al (2002) Reduction in the incidence of type 2 diabetes with lifestyle intervention or metformin. N Engl J Med 346:393-403 
4. Diabetes Prevention Program Research Group, Knowler WC, Fowler SE et al (2009) 10-year follow-up of diabetes incidence and weight loss in the Diabetes Prevention Program Outcomes Study. Lancet 374:1677-1686

5. Pan XR, Li GW, Hu YH et al (1997) Effects of diet and exercise in preventing NIDDM in people with impaired glucose tolerance. The Da Qing IGT and Diabetes Study. Diabetes Care 20:537-544

6. Li G, Zhang P, Wang J et al (2008) The long-term effect of lifestyle interventions to prevent diabetes in the China Da Qing Diabetes Prevention Study: a 20-year follow-up study. Lancet 371:1783-1789

7. Lindström J, Peltonen M, Eriksson JG et al (2013) Improved lifestyle and decreased diabetes risk over 13 years: long-term followup of the randomised Finnish Diabetes Prevention Study (DPS). Diabetologia 56:284-293

8. Ramachandran A, Snehalatha C, Mary S et al (2006) The Indian Diabetes Prevention Programme shows that lifestyle modification and metformin prevent type 2 diabetes in Asian Indian subjects with impaired glucose tolerance (IDPP-1). Diabetologia 49:289-297

9. Schwarz PE, Greaves CJ, Lindström J et al (2012) Nonpharmacological interventions for the prevention of type 2 diabetes mellitus. Nat Rev Endocrinol 8:363-373

10. Kantartzis K, Thamer C, Peter A et al (2009) High cardiorespiratory fitness is an independent predictor of the reduction in liver fat during a lifestyle intervention in non-alcoholic fatty liver disease. Gut 58:1281-1288

11. Häring H-U (2016) Novel phenotypes of prediabetes? Diabetologia 59:1806-1818

12. Ferrannini E (2014) Definition of intervention points in prediabetes. Lancet Diabetes Endocrinol 2:667-675

13. Kahn SE, Cooper ME, Del Prato S (2014) Pathophysiology and treatment of type 2 diabetes: perspectives on the past, present, and future. Lancet 383:1068-1083

14. Stefan N, Fritsche A, Schick F, Häring H-U (2016) Phenotypes of prediabetes and stratification of cardiometabolic risk. Lancet Diabetes Endocrinol 4:789-798

15. Stefan N, Staiger H, Wagner R et al (2015) A high-risk phenotype associates with reduced improvement in glycaemia during a lifestyle intervention in prediabetes. Diabetologia 58:2877-2884

16. Kitabchi AE, Temprosa M, Knowler WC et al (2005) Role of insulin secretion and sensitivity in the evolution of type 2 diabetes in the diabetes prevention program: effects of lifestyle intervention and metformin. Diabetes 54:2404-2414

17. Abdul-Ghani MA, Williams K, DeFronzo RA, Stern M (2007) What is the best predictor of future type 2 diabetes? Diabetes Care 30:1544-1548

18. Lyssenko V, Almgren P, Anevski D et al (2005) Predictors of and longitudinal changes in insulin sensitivity and secretion preceding onset of type 2 diabetes. Diabetes 54:166-174

19. de Mello VDF, Lindström J, Eriksson J et al (2012) Insulin secretion and its determinants in the progression of impaired glucose tolerance to type 2 diabetes in impaired glucose-tolerant individuals: the Finnish Diabetes Prevention Study. Diabetes Care 35:211-217

20. Perreault L, Pan Q, Mather KJ et al (2012) Effect of regression from prediabetes to normal glucose regulation on long-term reduction in diabetes risk: results from the Diabetes Prevention Program Outcomes Study. Lancet 379:2243-2251

21. Perreault L, Kahn SE, Christophi CA et al (2009) Regression from pre-diabetes to normal glucose regulation in the diabetes prevention program. Diabetes Care 32:1583-1588

22. Wareham NJ, Byrne CD, Williams R et al (1999) Fasting proinsulin concentrations predict the development of type 2 diabetes. Diabetes Care 22:262-270

23. Pfützner A, Kunt T, Hohberg C et al (2004) Fasting intact Proinsulin is a highly specific predictor of insulin resistance in type 2 diabetes. Diabetes Care 27:682-687
24. Mykkänen L, Zaccaro DJ, Hales CN et al (1999) The relation of proinsulin and insulin to insulin sensitivity and acute insulin response in subjects with newly diagnosed type II diabetes: the Insulin Resistance Atherosclerosis Study. Diabetologia 42:1060-1066

25. Bergman RN, Finegood DT, Kahn SE (2002) The evolution of $\beta$ cell dysfunction and insulin resistance in type 2 diabetes. Eur J Clin Investig 32:35-45

26. Kahn SE (2000) The importance of the beta-cell in the pathogenesis of type 2 diabetes mellitus. Am J Med 108(Suppl 6a):2S-8S

27. Hanley AJG, D'Agostino R, Wagenknecht LE et al (2002) Increased proinsulin levels and decreased acute insulin response independently predict the incidence of type 2 diabetes in the Insulin Resistance Atherosclerosis Study. Diabetes 51:1263-1270

28. Loopstra-Masters RC, Haffner SM, Lorenzo C et al (2011) Proinsulin-to-C-peptide ratio versus proinsulin-to-insulin ratio in the prediction of incident diabetes: the Insulin Resistance Atherosclerosis Study (IRAS). Diabetologia 54:3047-3054

29. Zethelius B, Byberg L, Hales CN et al (2003) Proinsulin and acute insulin response independently predict type 2 diabetes mellitus in men - report from 27 years of follow-up study. Diabetologia 46:20-26

30. Pfützner A, Pfützner AH, Larbig M, Forst T (2004) Role of intact Proinsulin in diagnosis and treatment of type 2 diabetes mellitus. Diabetes Technol Ther 6:405-412

31. Uusitupa M, Lindi V, Louheranta A et al (2003) Long-term improvement in insulin sensitivity by changing lifestyles of people with impaired glucose tolerance: 4-year results from the Finnish Diabetes Prevention Study. Diabetes 52:2532-2538

32. Yoshioka N, Kuzuya T, Matsuda A, Iwamoto Y (1989) Effects of dietary treatment on serum insulin and Proinsulin response in newly diagnosed NIDDM. Diabetes 38:262-266

33. Kahn SE, Halban PA (1997) Release of incompletely processed proinsulin is the cause of the disproportionate proinsulinemia of NIDDM. Diabetes 46:1725-1732

34. Røder ME, Porte D, Schwartz RS, Kahn SE (1998) Disproportionately elevated proinsulin levels reflect the degree of impaired B cell secretory capacity in patients with noninsulindependent diabetes mellitus. J Clin Endocrinol Metab 83:604-608

35. Larsson H, Ahrén B (1999) Relative Hyperproinsulinemia as a sign of islet dysfunction in women with impaired glucose tolerance. J Clin Endocrinol Metab 84:2068-2074

36. Hofsø D, Jenssen T, Bollerslev J et al (2011) Beta cell function after weight loss: a clinical trial comparing gastric bypass surgery and intensive lifestyle intervention. Eur J Endocrinol 164:231-238

37. Ramos JS, Dalleck LC, Borrani F et al (2016) The effect of different volumes of high-intensity interval training on proinsulin in participants with the metabolic syndrome: a randomised trial. Diabetologia 59:2308-2320

38. McCaffery JM, Jablonski KA, Franks PW et al (2011) TCF7L2 polymorphism, weight loss and proinsulin : insulin ratio in the diabetes prevention program. PLoS One 6:e21518

39. Kantartzis K, Machann J, Schick F et al (2011) Effects of a lifestyle intervention in metabolically benign and malign obesity. Diabetologia 54:864-868

40. Machann J, Thamer C, Stefan N et al (2010) Follow-up whole-body assessment of adipose tissue compartments during a lifestyle intervention in a large cohort at increased risk for type 2 diabetes. Radiology 257:353-363

41. Haupt A, Thamer C, Heni M et al (2010) Gene variants of TCF7L2 influence weight loss and body composition during lifestyle intervention in a population at risk for type 2 diabetes. Diabetes 59:747-750

42. Pfützner A, Kunt T, Langenfeld $M$ et al (2005) Clinical and laboratory evaluation of specific chemiluminescence assays for intact and total proinsulin. Clin Chem Lab Med 41:1234-1238

43. Matsuda M, DeFronzo RA (1999) Insulin sensitivity indices obtained from oral glucose tolerance testing: comparison with the euglycemic insulin clamp. Diabetes Care 22:1462-1470 
44. Szczepaniak LS, Nurenberg P, Leonard D et al (2005) Magnetic resonance spectroscopy to measure hepatic triglyceride content: prevalence of hepatic steatosis in the general population. Am J Physiol Endocrinol Metab 288:E462-E468

45. Lindström J, Louheranta A, Mannelin M et al (2003) The Finnish Diabetes Prevention Study (DPS): lifestyle intervention and 3-year results on diet and physical activity. Diabetes Care 26:3230-3236

46. Schellenberg ES, Dryden DM, Vandermeer B et al (2013) Lifestyle interventions for patients with and at risk for type 2 diabetes: a systematic review and meta-analysis. Ann Intern Med 159:543-551

47. Stefan N, Kantartzis K, Machann J et al (2008) Identification and characterization of metabolically benign obesity in humans. Arch Intern Med 168:1609-1616

48. Hotamisligil GS (2006) Inflammation and metabolic disorders. Nature 444:860-867

49. Stefan N, Hennige AM, Staiger H et al (2006) $\alpha_{2}$-Heremans-Schmid glycoprotein/fetuin-A is associated with insulin resistance and fat accumulation in the liver in humans. Diabetes Care 29:853-857

50. Snehalatha C, Mary S, Selvam S et al (2009) Changes in insulin secretion and insulin sensitivity in relation to the glycemic outcomes in subjects with impaired glucose tolerance in the Indian
Diabetes Prevention Programme-1 (IDPP-1). Diabetes Care 32: 1796-1801

51. Ahrén B (2005) Type 2 diabetes, insulin secretion and beta-cell mass. Curr Mol Med 5:275-286

52. Fritsche A, Madaus A, Stefan N et al (2002) Relationships among age, Proinsulin conversion, and $\beta$-cell function in nondiabetic humans. Diabetes 51:S234-S239

53. Vangipurapu J, Stančáková A, Kuulasmaa T et al (2015) Both fasting and glucose-stimulated proinsulin levels predict hyperglycemia and incident type 2 diabetes: a population-based study of 9 , 396 Finnish men. PLoS One 10:e0124028

54. Nijpels G, Popp-Snijders C, Kostense PJ et al (1996) Fasting proinsulin and 2-h post-load glucose levels predict the conversion to NIDDM in subjects with impaired glucose tolerance: the Hoorn study. Diabetologia 39:113-118

55. Haffner SM, Mykkänen L, Valdez RA et al (1994) Disproportionately increased proinsulin levels are associated with the insulin resistance syndrome. J Clin Endocrinol Metab 79:1806-1810

56. American Diabetes Association (2016) Prevention or delay of type 2 diabetes. Diabetes Care 39(Suppl 1):S36-S38 